\title{
Label-free immunodetection of the cancer biomarker CA125 using high- $\Delta n$ liquid crystals
}

Hui-Wen Su

Yun-Han Lee

Mon-Juan Lee

Yi-Chiang Hsu

Wei Lee

\section{SPIE.}




\title{
Label-free immunodetection of the cancer biomarker CA125 using high- $\Delta n$ liquid crystals
}

\author{
Hui-Wen Su, ${ }^{a}$ Yun-Han Lee, ${ }^{a}$ Mon-Juan Lee, ${ }^{b, *}$ Yi-Chiang Hsu, ${ }^{c}$ and Wei Lee ${ }^{a, \star}$ \\ ${ }^{a}$ National Chiao Tung University, College of Photonics, Tainan 71150, Taiwan \\ ${ }^{b}$ Chang Jung Christian University, Department of Bioscience Technology, Tainan 71101, Taiwan \\ ${ }^{\circ}$ Chang Jung Christian University, Graduate Institute of Medical Sciences, Tainan 71101, Taiwan
}

\begin{abstract}
A label-free and array-based optical liquid-crystal (LC) immunodetection technique for the detection of CA125 antigen, a protein biomarker most frequently used for ovarian cancer detection, was demonstrated with a nematic LC with larger birefringence $(\Delta n)$ to promote sensitivity in detecting biomolecules. The LC-based immunodetection offers an alternative and sensitive approach for the detection of biomarker proteins, with the potential of replacing conventional immunoassays used in biochemical studies and clinical laboratories. $\odot$ The Authors. Published by SPIE under a Creative Commons Attribution 3.0 Unported License. Distribution or reproduction of this work in whole or in part requires full attribution of the original publication, including its DOI. [DOI: 10.1117/1.JBO.19.7.077006]
\end{abstract}

Keywords: liquid-crystal-based immunodetection; cancer biomarker; CA125; label-free immunodetection.

Paper 140214SSR received Apr. 3, 2014; revised manuscript received Jun. 10, 2014; accepted for publication Jun. 23, 2014; published online Jul. 23, 2014.

\section{Introduction}

Immunodetection of cancer biomarkers is an essential procedure in cancer detection and diagnosis. Currently, the most widely used method is sandwich assays, in which the strength of chemiluminescence released from labeled molecules is analyzed to quantify the level of cancer biomarkers.

CA125 is a mucin-like glycoprotein with a molecular weight over $200 \mathrm{kDa}$, which was first discovered over 30 years ago using the OC125 monoclonal antibody, ${ }^{1}$ providing the basis for assays to detect and monitor the progression of epithelial ovarian cancer. The commercially available CA125 immunoassay has a detection limit of $15 \mathrm{U} / \mathrm{ml}$, which is sufficient to detect the generally accepted threshold level of CA125 when the disease is present $(35 \mathrm{U} / \mathrm{ml}) .{ }^{2}$ Due to the importance of early diagnosis of cancer, various immunoassays for the detection of CA125 cancer biomarker have been reported, including enzyme-linked immunosorbent assay, ${ }^{3}$ electrochemical immunoassay, ${ }^{4-7}$ and colorimetric immunoassay. ${ }^{8}$ These traditional label-based immunoassays are often complicated, timeconsuming, with the high cost of labeling antibodies. As such, development of label-free immunodetection techniques is highly desired to reduce the cost of detection systems as well as to simplify cancer screening procedures.

Since Abbott's group reported the use of the liquid crystal (LC) 5CB (4-pentyl-4'-cyanobiphenyl) as a sensing element to detect biomolecules, ${ }^{9}$ LC-based biosensors have received much attention. ${ }^{10-16}$ It is known that biomolecules immobilized or adsorbed on solid surfaces may trigger the reorientation of LC molecules, and the effect depends on the concentration of biomolecules. ${ }^{10}$ When the reorientation of LCs occurs, ${ }^{17}$ optical responses can be observed under a polarized optical microscope, whose two polarizers are placed after the light source and before the eyepiece or camera through the light passage. To explain the reorientation, the molecular theory of surface tension ${ }^{18}$ can be

*Address all correspondence to: Mon-Juan Lee, E-mail: mjlee@mail.cjcu.edu .tw; Wei Lee, E-mail: wlee@nctu.edu.tw adopted. In this theory, the critical surface tension $\gamma_{\mathrm{c}}$ is introduced as the criterion for homeotropic alignment (LC molecules being perpendicular to the substrate) or homogeneous alignment (LC molecules being parallel to the substrate), such that if $\gamma_{\mathrm{lc}}>\gamma_{\mathrm{c}}$ then the liquid crystalline material shows homeotropic alignment, and if $\gamma_{\mathrm{lc}} \ll \gamma_{\mathrm{c}}$ then the liquid crystalline material shows homogeneous alignment. As the surface tension $\gamma_{\mathrm{lc}}$ drops below the critical value $\left(\gamma_{\mathrm{c}}\right)$, the orientation deviates from perpendicular alignment and may take a tilt angle.

Currently, LC-based biosensing is applied in the detection of enzymatic reactions, antibody-antigen immunoreactions, DNA hybridization, and so on. ${ }^{9,11,12}$ However, 5CB has seemingly been the only choice and little attention has been paid to the selection of LCs or to how the birefringence (i.e., optical anisotropy) $\Delta n$ is correlated with the sensitivity in this type of label-free immunodetection. ${ }^{13-16}$

In this work, we demonstrate that, in the detection of biomolecules, higher sensitivity can be well achieved through a more significant phase retardation using an LC mixture of larger birefringence material designated $\operatorname{HDN}(\Delta n=0.333)$ instead of the commonly applied nematogen $5 \mathrm{CB}(\Delta n=0.179) .{ }^{9-16} \mathrm{CA} 125$, a biomarker for several types of cancer, such as ovarian cancer and breast cancer, is reacted with its antibodies immobilized on glass slides coated with alignment layers, followed by detection with LCs in the absence of labeled molecules (i.e., no extra labeling, such as fluorescence, is used). This LC-based immunodetection was used to study the correlation between CA125 detection sensitivity and the optical properties of LCs, so as to develop a simple, highly sensitive, and label-free LC-based immune biosensor.

\section{Materials and Methods}

\subsection{Materials}

The surfactant dimethyloctadecyl[3-(trimethoxysilyl)propyl] ammonium chloride (DMOAP) as the vertical alignment agent $^{19}$ was purchased from Sigma-Aldrich (St. Louis, Missouri). Two LCs, a eutectic nematic mixture designated 
HDN (birefringence $\Delta n=0.333$ at a wavelength of $589.3 \mathrm{~nm}$ and a temperature of $20^{\circ} \mathrm{C}$ ) and the cyanobiphenyl single compound $5 \mathrm{CB}\left(\Delta n=0.179\right.$ at $589.3 \mathrm{~nm}$ and $\left.25^{\circ} \mathrm{C}\right)$, were provided by HCCH (Jiangsu Hecheng Display Technology Co., Ltd.) and Daily Polymer, respectively. Recombinant human CA125 and bovine serum albumin (BSA) were received from R\&D Systems and Sigma-Aldrich, respectively. Antibodies against CA125, spermidine/spermine $\mathrm{N}^{1}$-acetyl transferase (SSAT) and $\beta$-catenin, were manufactured by Santa Cruz Biotechnology.

\subsection{Preparation of DMOAP-Coated Glass Slides}

The glass slides were immersed in an aqueous solution containing Alconox ${ }^{\circledR}$ detergent, then rinsed two times with deionized (DI) water to remove excess detergent and immersed in $99 \%$ ethanol. Alconox ${ }^{\circledR}$ detergent is for cleaning glass substrate surface. All steps mentioned above were performed in an ultrasonic bath for $15 \mathrm{~min}$ at room temperature (RT). Subsequently, the glass slides were dried under a stream of nitrogen and then baked at $74^{\circ} \mathrm{C}$ for $15 \mathrm{~min}$. The cleaned glass slides were immersed in an aqueous solution containing $1 \%(\mathrm{v} / \mathrm{v})$ DMOAP for $15 \mathrm{~min}$ at RT, rinsed with DI water for $1 \mathrm{~min}$ to remove excess DMOAP solution, dried under a stream of nitrogen, and finally heated at $100^{\circ} \mathrm{C}$ for $15 \mathrm{~min}$.

\subsection{Immunodetection}

Prior to use, protein solutions with desired concentrations were prepared by dilution with DI water. CA125 mouse-antibody was immobilized on the DMOAP-coated glass slide at $1 \mu \mathrm{l} /$ droplet in an array format. After drying overnight at RT, 20- $\mu$ 1 CA125 antigen at desired concentrations is dropped on the immobilized antibody array and the substrate was covered with a clean cover glass to form a cell. CA125 antigen and antibody were allowed to react for $0.5 \mathrm{~h}$ at RT. Afterward, the cover glass was removed, and the glass slides were rinsed twice with DI water and dried on a hot plate at $30^{\circ} \mathrm{C}$ for $2 \mathrm{~min}$ to remove excess water.

\subsection{Assembly of LC Cells}

An LC optical cell was assembled by pairing two DMOAPcoated glass slides, one without any further treatment (referred to as the upper substrate) and the other with analyte immobilized in an array format (referred to as the lower substrate). Spacers mixed with ethanol were dispensed on the four corners of the lower substrate and then allowed to dry at RT for $5 \mathrm{~min}$. The lower substrate was then covered with an upper substrate, sealed with AB glue to form an LC cell, and dried at RT for another $2 \mathrm{~h}$. Finally, the LC cell was filled with LC by capillary action. The optical texture of the LC cell was observed under a microscope with crossed polarizers in the transmission mode with an exposure time of $14.84 \mathrm{~ms}$, which is the default value for the image capture system. The polarized optical microscope (Olympus, Tokyo, Japan, BX51) is equipped with a halogen lightbulb as the light source (Philips projection lamp type 7023, $12 \mathrm{~V}$, $100 \mathrm{~W}$ ). Figure 1 depicts the sample preparation procedure of the LC-based immunodetection.

\subsection{Quantitative Analysis of LC-based Immunodetection}

When the immunocomplex of CA125 is generated on the surface of a substrate, the surface tension decreases. In this case, the polarized light can be transmitted through the second polarizer and collected as an optical signal. The fraction of light passing through the second polarizer is directly related to a phase retardation described by: ${ }^{20}$ (a)

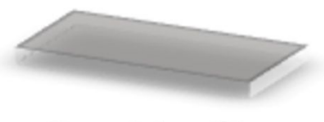

Cleaned glass slide

(c)

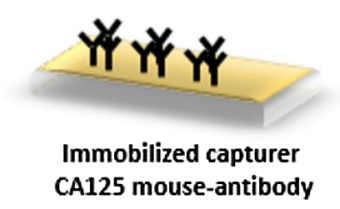

(e)

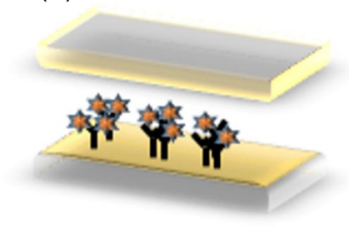

Assembled LCs cell (b)

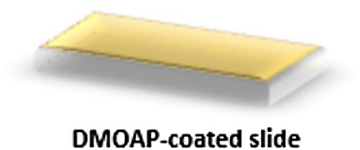

(d)

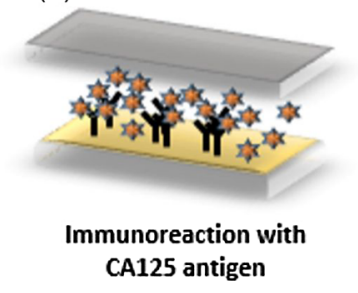

(f)

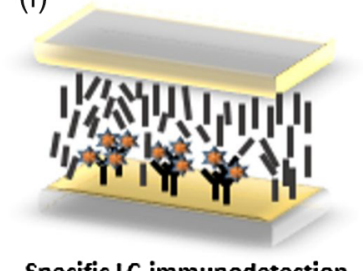

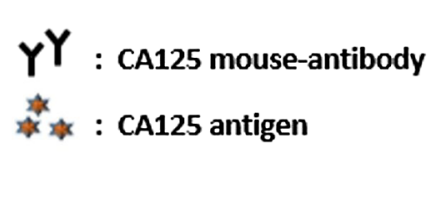

Fig. 1 The procedure of liquid crystal (LC)-immunodetection includes (a) cleaning of glass slides, (b) coating of dimethyloctadecyl[3-(trimethoxysilyl)propyl]ammonium chloride alignment layer, (c) immobilization of CA125 mouse antibody, (d) immunoreaction with CA125 antigen, (e) assembly of LC cell, and $(f)$ infiltration of LC into the cell. 
$\delta=\frac{2 \pi \cdot d \Delta n}{\lambda}$

where $d$ is the thickness of the disturbed LCs layer, $\lambda$ is the wavelength, and $\Delta n$ is the optical birefringence. This indicates that with a higher birefringence one can obtain a higher phase retardation. When placed between crossed polarizers, the intensity of the light emerging from the second polarizer can be written as

$I \propto I_{0} \sin ^{2} \delta$.

Equation (2) connotes that, with higher phase retardation, a higher signal yield can be obtained.

The freeware GIMP, which is a digital image analysis software, was exploited to quantify the optical textures. The R, G, and $\mathrm{B}$ values of each pixel in the circular signal region are summed and averaged. We refer to the obtained values as the relative intensity. For each concentration condition, at least six samples are used in the average. The corresponding standard deviations were taken as errors.

\subsection{Fluorescence Immunoassay for the CA125 Antigen}

For comparison, CA125 antigen at various concentrations was immobilized with $1 \mu \mathrm{l}$ /droplet on a 24 -well cell culture plate. After drying at RT for $3 \mathrm{~h}, 5 \mathrm{mg} / \mathrm{ml}$ BSA was reacted with CA125 antigen for $1.5 \mathrm{~h}$ to block nonspecific immobilization of undesired biomolecules. Each well was rinsed once with the phosphate-buffered saline with Tween 20 (PBST) buffer, which contains $3.2 \mathrm{mM} \mathrm{Na} \mathrm{HPO}_{4}, 0.5 \mathrm{mM} \quad \mathrm{KH}_{2} \mathrm{PO}_{4}$, $1.3 \mathrm{mM} \mathrm{KCl}, 135 \mathrm{mM} \mathrm{NaCl}$, and $0.05 \%$ (v/v) Tween 20, $\mathrm{pH}$ 7.4. After another $1.5 \mathrm{~h}$, IRDye-labeled secondary antibody (LI-COR, Inc., Lincoln, Nebraska) with a dilution ratio of 1:5000 was added to each well to react with the CA125 antigen-antibody complex at RT for $0.5 \mathrm{~h}$. Each well was rinsed once with PBST buffer and twice with PBS prior to fluorescence detection. The fluorescence signal and image were acquired with an Odyssey® Infrared Imaging System (LI-COR, Inc., Lincoln, Nebraska) equipped with a solid-state diode laser at $785 \mathrm{~nm}$.

\section{Results}

\subsection{Detection of $B S A$ with $H D N$ or $5 C B$}

We hypothesized that LCs of high birefringence, such as HDN, may enhance the sensitivity of protein detection by generating a more significant phase retardation in the presence of biomolecules with respect to LCs with lower birefringence, such as 5CB. We tested this hypothesis by immobilizing BSA on the DMOAP alignment layer, followed by detection with HDN or $5 \mathrm{CB}$. The optical textures and the corresponding total intensities of $5 \mathrm{CB}$ or $\mathrm{HDN}$ in contact with various concentrations of BSA were shown in Figs. 2 and 3, respectively. It is particularly clear from the quantified data (Fig. 3) that, for a given BSA concentration, HDN gives rise to a more pronounced dark-to-bright optical response than does $5 \mathrm{CB}$, indicating that the higher sensitivity can be achieved by using HDN in LC-based biosensing. Note that for the captured image with lower signal yields in Fig. 2(c), the green dashed circle indicates the region of the dropped analyte BSA. With this finding in mind, the following study investigates the use of HDN as the major sensing element in LC-based immunodetection.

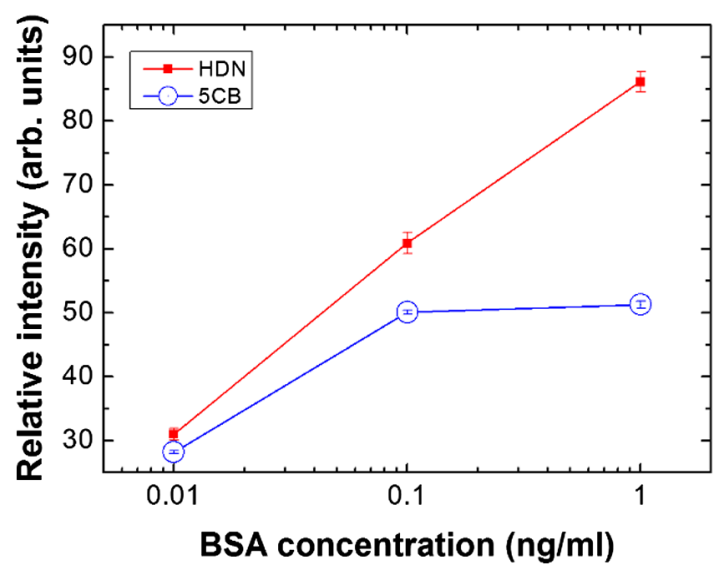

Fig. 3 Comparison between the relative intensities of optical textures of $5 \mathrm{CB}$ and HDN in contact with bovine serum albumin of various concentrations.
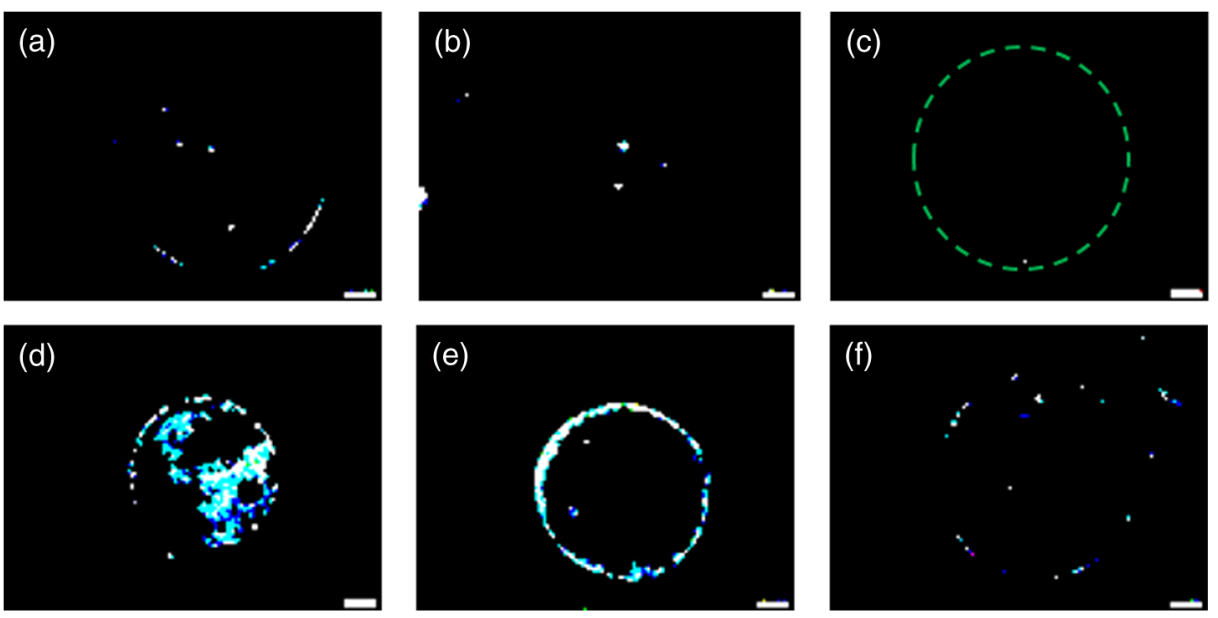

Fig. 2 Optical textures of $(a-c) 5 C B$ and (d-f) $H D N$ in the presence of $(a, d) 1 \mathrm{ng} / \mathrm{ml},(b, e) 0.1 \mathrm{ng} / \mathrm{ml}$, or (c, f) $0.01 \mathrm{ng} / \mathrm{ml} \mathrm{BSA}$. Bar, $200 \mu \mathrm{m}$. 


\subsection{Immunodetection of the Cancer Biomarker CA125 by HDN}

The immunodetection of cancer biomarker CA125 by HDN was performed by immobilizing the anti-CA125 antibody on a glass substrate coated with DMOAP. After interaction with the CA125 antigen, formation of the immunocomplex was detected with HDN under a crossed polarizing microscope. In order to find the proper concentration of the anti-CA125 antibody and to reduce background disturbance, we first examined the optical appearance of $\mathrm{HDN}$ when an antibody array containing $1 \mu \mathrm{l} /$ spot of $0.1-\mu \mathrm{g} / \mathrm{ml}$ and $0.01-\mu \mathrm{g} / \mathrm{ml}$ anti-CA125 antibody was reacted with $0.01,0.1,1$, or $10 \mu \mathrm{g} / \mathrm{ml}$ CA125 antigen. As shown in Figs. 4 and 5, the presence of CA125 antigen-antibody immunocomplex disrupted the alignment of LC and changed the optical texture of HDN. However, the difference between the optical textures of HDN at different CA125 antigen concentrations, such as 1 and $10 \mu \mathrm{g} / \mathrm{ml}$, was insignificant when the antibody concentration is $0.01 \mu \mathrm{g} / \mathrm{ml}$. This implies the concentration may be insufficient for the immunodetection of the CA125 antigen in the range of 0.01 to $10 \mu \mathrm{g} / \mathrm{ml}$. Nonetheless, when the concentration of the anti-CA125 antibody was increased to $0.1 \mu \mathrm{g} / \mathrm{ml}$, the optical response of HDN was dependent on the antigen concentration, leading to a brighter optical texture when a higher concentration of the CA125 antigen was present [see Figs. 4(a)-4(d)]. Note that a bright ring may appear in the optical texture due to the so-called coffee-ring effect. This is a result of the slow drying process in which the colloidal suspension in the droplet was pushed to the peripheral edge, forming a circular ring after the droplet was fully dried. ${ }^{21}$

\subsection{Specificity of LC-based Immunodetection}

To examine the specificity of LC-based immunodetection, the CA125 antigen was reacted with a nonspecific antibody array of $0.1-\mu \mathrm{g} / \mathrm{ml}$ anti-SSAT or anti- $\beta$-catenin. As shown in Fig. 6, in the absence of anti-CA125 antibodies, immunocomplex was not formed, and the optical appearance of HDN remained dark. This indicates that the nonspecific anti-SSAT or anti- $\beta$-catenin antibodies were unable to complex with the CA125 antigen and thus cannot significantly disrupt LC. In other words, the orientation of LC molecules remained homeotropic.

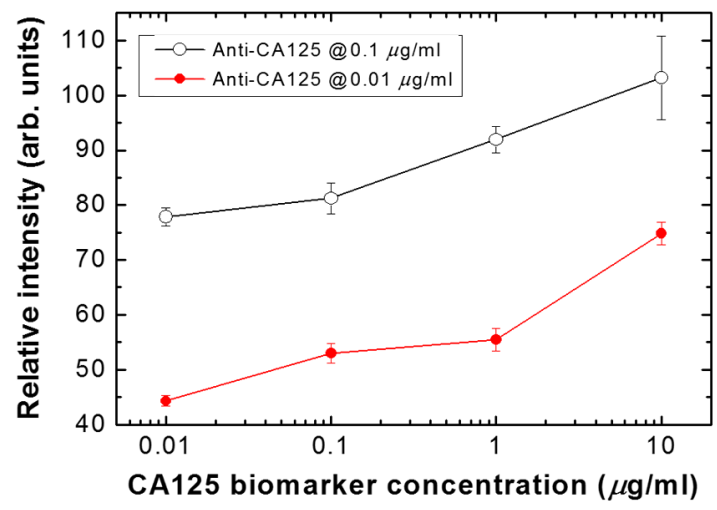

Fig. 5 Relative intensities of optical textures in LC-immunoreaction with HDN at various CA125 concentrations.

\subsection{Immunofluorescence Detection of CA125}

A parallel fluorescence immunoassay was performed in a similar array format as in LC-based immunodetection to compare detection sensitivity. In the fluorescence immunoassay, 4-, 5-, or $10-\mu \mathrm{g} / \mathrm{ml} \mathrm{CA} 125$ antigen was immobilized on a $24-$ well cell culture plate and reacted with $1-\mu \mathrm{g} / \mathrm{ml}$ anti-CA125 antibody, followed by detection with fluorescence-labeled secondary antibodies [Fig. 7(a)]. Quantitative results suggest that the relative fluorescence intensity increased with increasing concentrations of CA125 antigen [Fig. 7(b)]. As an immunofluorescence signal was barely detectable at $4 \mu \mathrm{g} / \mathrm{ml}$, we conclude that the detection limit of fluorescence immunoassay for CA125 was 4 to $5 \mu \mathrm{g} / \mathrm{ml}$ in this comparative study.

\section{Discussion}

\subsection{Optimal Concentration of CA125 Antibody}

Prior to immunodetection, it is necessary to choose a suitable anti-CA125 antibody concentration to ensure that not only the CA125 antigen is sufficiently bound to the antibody, but also the optical texture of LCs is not affected by the CA125 antibodies in the absence of the CA125 antigen. We found that when the concentration of anti-CA125 antibody was $0.1 \mu \mathrm{g} / \mathrm{ml}$, which corresponds to $1 \mu \mathrm{g} / \mathrm{spot}$ in the array, the change in the optical texture of HDN was correlated to the amount of CA125 present, suggesting that an antibody concentration of
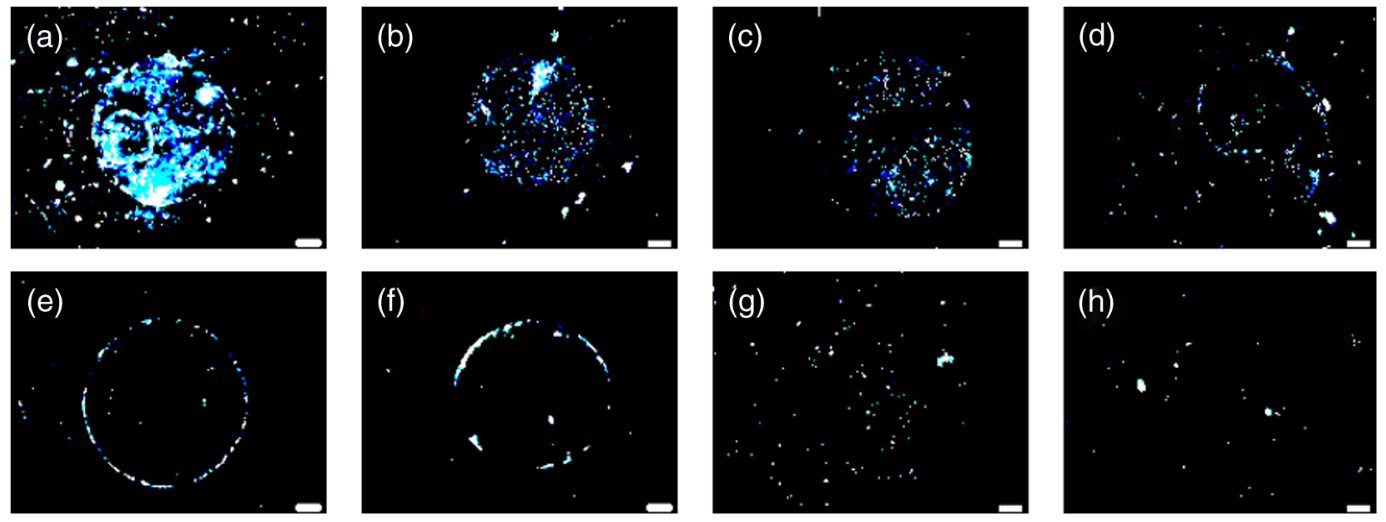

Fig. 4 Optical textures of HDN in the presence of (a-d) 0.1 or (e-h) $0.01 \mu \mathrm{g} / \mathrm{ml}$ anti-CA125 antibody complexed with $(\mathrm{a}, \mathrm{e}) 10,(\mathrm{~b}, \mathrm{f}) 1,(\mathrm{c}, \mathrm{g}) 0.1$, or $(\mathrm{d}, \mathrm{h}) 0.01 \mu \mathrm{g} / \mathrm{ml}$ CA125 protein. Bar, $200 \mu \mathrm{m}$. 

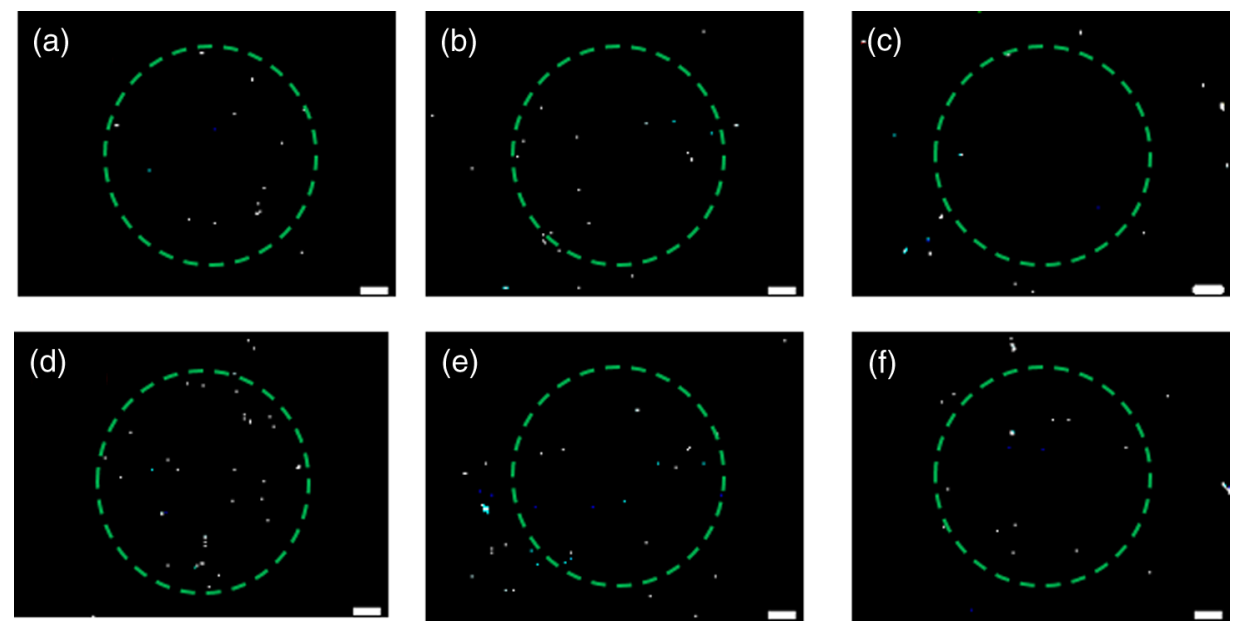

Fig. 6 Optical textures of HDN in the presence of $0.1 \mu \mathrm{g} / \mathrm{ml}(\mathrm{a}-\mathrm{c})$ anti-SSAT antibody or (d-f) anti- $\beta$-catenin antibody, which was reacted with (a, d) 0 , (b, e) 0.1 , or (c, f) $1 \mu \mathrm{g} / \mathrm{ml}$ CA125 protein. Bar, $200 \mu \mathrm{m}$.

$0.1 \mu \mathrm{g} / \mathrm{ml}$ is suitable for the design of array-based immunodetection with LCs [Figs. 4(a)-4(d)].

\subsection{Detection Sensitivity of LC-Based Immunoassay}

At a CA125 antigen concentration of $0.01 \mu \mathrm{g} / \mathrm{ml}$, the change in the optical texture can barely be observed, implying that the

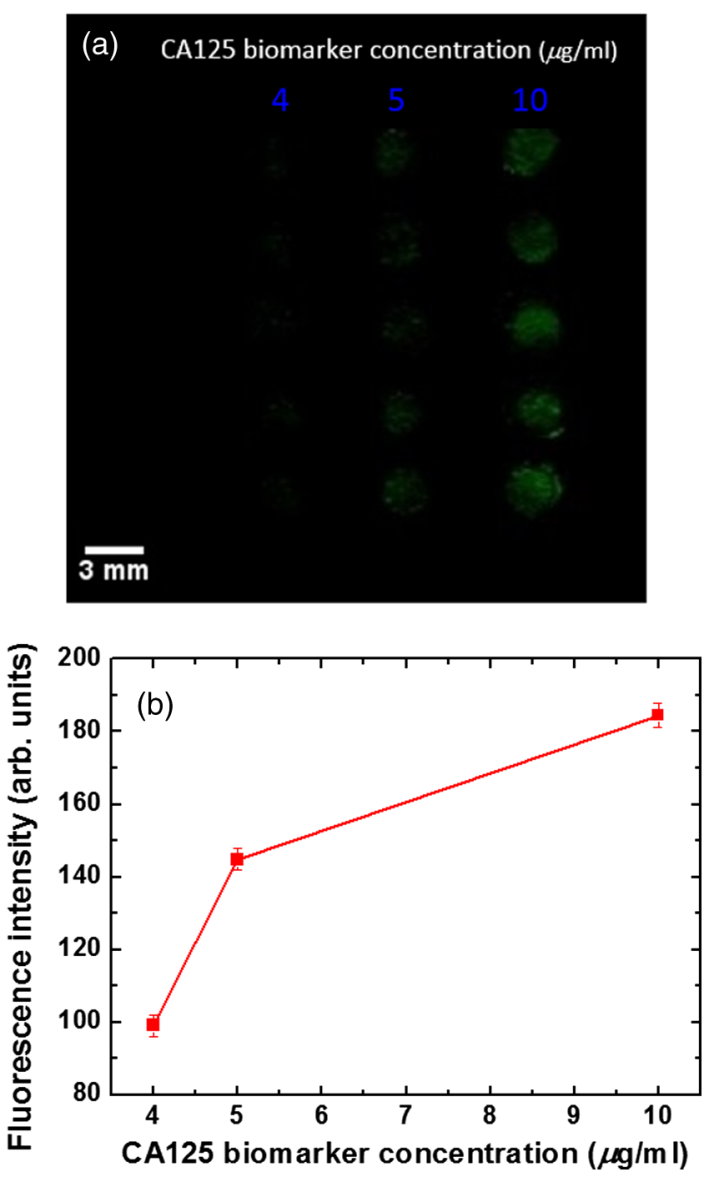

Fig. 7 (a) Fluorescence image and (b) relative fluorescence intensity of various concentrations of the cancer biomarker CA125 detected by anti-CA125 antibodies and fluorescence-labeled secondary antibodies in a fluorescence immunoassay.
Table 1 Comparison between LC-based and fluorescence immunossays.

\begin{tabular}{lccc}
\hline \multicolumn{1}{c}{$\begin{array}{c}\text { Sensing } \\
\text { platform }\end{array}$} & $\begin{array}{c}\text { Detection limit } \\
\text { for CA125 } \\
(\mu \mathrm{g} / \mathrm{ml})\end{array}$ & $\begin{array}{c}\text { Range of } \\
\text { detection for } \\
\text { CA125 }(\mu \mathrm{g} / \mathrm{ml})^{\mathrm{a}}\end{array}$ & Characteristics \\
\hline $\begin{array}{l}\text { LC-based } \\
\text { immunodetection }\end{array}$ & $0.01-0.1$ & $0.01-250$ & $\begin{array}{c}\text { Simplified label- } \\
\text { free procedure }\end{array}$ \\
$\begin{array}{l}\text { Fluorescence } \\
\text { immunoassay }\end{array}$ & $4-5$ & $4-250$ & $\begin{array}{c}\text { Complicate } \\
\text { label-based } \\
\text { procedure }\end{array}$ \\
\hline
\end{tabular}

${ }^{\text {aT }}$ The upper limit of $250 \mu \mathrm{g} / \mathrm{ml}$ is the highest concentration of recombinant CA125 provided by R\&D Systems.

detection limit of LC-based immunodetection was within the range of 0.01 to $0.1 \mu \mathrm{g} / \mathrm{ml}$. In comparison, the detection limit of an array-based fluorescence immunoassay carried out in this study was 4 to $5 \mu \mathrm{g} / \mathrm{ml}$, which was much higher than that of the LC-based immunoassay. These results suggest that the label-free immunodetection with LCs is a promising alternative to conventional label-based immunoassays. A comparison between the LC-based and fluorescence immunoassays was summarized in Table 1. It is worth mentioning that the optical textures are still the same 1 year after the experiment. We thus consider the LC-based biosensor is of good stability for immunoassay.

\section{Conclusions}

In this study, a label-free and array-based optical LC-immunodetection technique was developed. Our results suggest that LCs with larger birefringence, such as HDN, are more sensitive in detecting biomolecules compared with the conventional LC $5 \mathrm{CB}$. In addition, choosing an appropriate antibody concentration in the design of the antibody array is critical in determining the sensitivity of LC-based immunodetection. When interacted with $0.1 \mu \mathrm{g} / \mathrm{ml}$ anti-CA125 antibody, higher concentrations of the cancer biomarker CA125 resulted in greater disruption of the optical texture of HDN, giving rise to a brighter optical response. Such a disruption was not seen when the anti-CA125 antibody was replaced by nonspecific ones. These results connote 
that LC-based immunodetection is specific, and the change in its optical texture is correlated with the analyte concentration. The detection limit of LC-based immunoassay for CA125 is within 0.01 to $0.1 \mu \mathrm{g} / \mathrm{ml}$, significantly lower than the 4 to $5 \mu \mathrm{g} / \mathrm{ml}$ of fluorescence immunoassay. Label-free immunodetection techniques based on birefringent LCs have the potential of detecting a wide range of biomolecules and should be further pursued as one in addition to conventional immunoassays ubiquitously used in biochemical studies and clinical laboratories.

\section{Acknowledgments}

This work was financially supported in part by Grant Nos. NSC 101-2112-M-009-018-MY3 and NSC 101-2314-B-309-001MY3 from the Ministry of Science and Technology, Taiwan.

\section{References}

1. R. C. Bast, Jr. et al., "Reactivity of a monoclonal antibody with human ovarian carcinoma," J. Clin. Invest. 68(5), 1331-1337 (1981).

2. J. Das and S. O. Kelley, "Protein detection using arrayed microsensor chips: tuning sensor footprint to achieve ultrasensitive readout of CA125 in serum and whole blood," Anal. Chem. 83(4), 1167-1172 (2011).

3. N. Scholler et al., "Bead-based ELISA for validation of ovarian cancer early detection markers," Clin. Cancer Res. 12(7), 2117-2124 (2006).

4. A. Guo et al., "An ultrasensitive enzyme-free electrochemical immunosensor for CA125 using Au@Pd core-shell nanoparticles as labels and platforms for signal amplification," J. Mater. Chem. B 1(33), 40524058 (2013).

5. J. Lin and H. Ju, "Electrochemical and chemiluminescent immunosensors for tumor markers," Biosens. Bioelectron. 20(8), 1461-1470 (2005).

6. D. Sok et al., "Novel fluoroimmunoassay for ovarian cancer biomarker CA-125," Anal. Bioanal. Chem. 393(5), 1521-1523 (2009).

7. D. Tang, R. Yuan, and Y. Chai, "Electrochemical immuno-bioanalysis for carcinoma antigen 125 based on thionine and gold nanoparticlesmodified carbon paste interface," Anal. Chim. Acta 564(2), 158-165 (2006).

8. Y. Yin et al., "Colorimetric immunoassay for detection of tumor markers," Int. J. Mol. Sci. 11(12), 5077-5094 (2010).

9. S. R. Kim and N. L. Abbott, "Rubbed films of functionalized bovine serum albumin as substrates for the imaging of protein-receptor interactions using liquid crystals," Adv. Mater. 13(19), 1445-1449 (2001).

10. C.-Y. Xue and K.-L. Yang, "Dark-to-bright optical responses of liquid crystals supported on solid surfaces decorated with proteins," Langmuir 24(2), 563-567 (2008).

11. B. H. Clare and N. L. Abbott, "Orientations of nematic liquid crystals on surfaces presenting controlled densities of peptides: amplification of protein-peptide binding events," Langmuir 21(14), 6451-6461 (2005).

12. C.-H. Chen and K.-L. Yang, "Detection and quantification of DNA adsorbed on solid surfaces by using liquid crystals," Langmuir 26(3), 1427-1430 (2010).

13. A. D. Price and D. K. Schwartz, "DNA hybridization-induced reorientation of liquid crystal anchoring at the nematic liquid crystal/aqueous interface," J. Am. Chem. Soc. 130(26), 8188-8194 (2008).

14. H. Tan et al., "Signal-enhanced liquid-crystal DNA biosensors based on enzymatic metal deposition," Angew. Chem. Int. Ed. 49(46), 8608-8611 (2010).
15. S. Yang et al., "Gold nanoparticle based signal enhancement liquid crystal biosensors for DNA hybridization assays," Chem. Commun. 48(23), 2861-2863 (2012).

16. S. Yang et al., "Label-free liquid crystal biosensor based on specific oligonucleotide probes for heavy metal ions," Anal. Chem. 85(1), 14-18 (2013).

17. V. K. Gupta et al., "Optical amplification of ligand-receptor binding using liquid crystals," Science 279(5359), 2077-2080 (1998).

18. T.-S. Wong et al., "Nanochromatography driven by the coffee ring effect," Anal. Chem. 83(6), 1871-1873 (2011).

19. F. J. Kahn, "Orientation of liquid crystals by surface coupling agents," Appl. Phys. Lett. 22(8), 386-388 (1973).

20. D. Andrienko, "Introduction to Liquid Crystals," http://www2.mpipmainz.mpg.de/ andrienk/teaching/IMPRS/liquid_crystals.pdf (14 September 2006).

21. T. Kohki et al., Chapter 3.7 in Alignment Technologies and Applications of Liquid Crystal Devices, pp. 91-93, Taylor \& Francis, New York (2005).

Hui-Wen Su received her BS degree in biomedical engineering from I-Shou University, Kaohsiung, Taiwan, in 2012 and her MS degree in imaging and biomedical photonics from the College of Photonics, National Chiao Tung University, Tainan, Taiwan, in 2014. Her focus of research has been on liquid-crystal-based immunodetection for cancer biomarkers.

Yun-Han Lee received his MS degree in physics from National Taiwan University, Taipei, Taiwan, in 2013. He is currently a PhD student at National Chiao Tung University. His research areas include the structural analysis of disclination of liquid-crystalline materials and the application of liquid crystals in display technology and biosensing devices.

Mon-Juan Lee received her MS degree in chemical engineering in 2000 and PhD degree in life science in 2006 from National Tsing Hua University, Hsinchu, Taiwan. She is currently an associate professor of the Department of Bioscience Technology with joint affiliation in the Graduate Institute of Medical Sciences at Chang Jung Christian University, Tainan, Taiwan. Her research interests include stem cell and bone biology, nanobiotechnology, as well as LC-based biomedical applications.

Yi-Chiang Hsu received his MS and PhD degrees in medical sciences in 1996 and 2007 both from Taipei Medical University, Taipei, Taiwan. He is currently an associate professor of the Graduate Institute of Medical Sciences at Chang Jung Christian University, Tainan, Taiwan. His research interests include biomedicine and nanomaterials.

Wei Lee earned his MS degree in electro-optical engineering from National Chiao Tung University (NCTU), Taiwan, in 1987 and his $\mathrm{PhD}$ degree in physics from the University of Alabama at Birmingham, Alabama, in 1993. He is currently the director of the Institute of Imaging and Biomedical Photonics, College of Photonics, NCTU, and the president of Taiwan Liquid Crystal Society. His primary research interests focus on liquid-crystal (LC) optics and photonics including LC immunoassay applications. 